\title{
Tiny Houses als Symbole von Selbstverwirklichung und Solidarität auf der Prämisse von Selbstaktivierung
}

\author{
Jan Finzi · Janine Kuhnt
}

Eingegangen: 24. Februar 2020 / Angenommen: 2. Mai 2021 / Online publiziert: 2. November 2021

(C) Der/die Autor(en) 2021

Zusammenfassung Auf der Grundlage einer Analyse der Selbstpräsentation von Akteuren der Tiny-House-Bewegung, können Tiny Houses einerseits als symbolisierte Selbstverwirklichung eines Milieus, das ressourcenbedingt eine (eigene) Norm des Wohnens konstituiert, verstanden werden und andererseits als Ausdruck von Solidarität mit von Wohnungsnot Betroffenen, die aus der Selbstaktivierung einer engagierten und solidarischen Zivilgesellschaft hervorgeht. Sowohl das ressourcenbedingte Setzen einer Norm des Wohnens auf kleinstem Raum als auch die Solidaritätsleistung engagierter Personen - mit und für von Wohnungsnot Betroffenen - verdeutlichen, dass Engagement zur Linderung von Wohnungsnot (durch Tiny Houses) voraussetzungsvoll und an die Selbstaktivierungskräfte zivilgesellschaftlicher Akteur*innen gebunden ist.

Schlüsselwörter Engagement · Zivilgesellschaft · Tiny-House-Bewegung · Wohnungsnot $\cdot$ Selbstverwirklichung

\footnotetext{
Jan Finzi, M.A. $(\varangle)$

Fachgebiet Qualitative Forschungsmethoden und strategische Kommunikation für Gesundheit, Inklusion und Teilhabe, Technische Universität Dortmund, Emil-Figge-Str. 50, 44227 Dortmund, Deutschland

E-Mail: jan.finzi@tu-dortmund.de

Janine Kuhnt, M.A.

Institut für Erziehungswissenschaft, Lehrstuhl für Sozialpädagogik und außerschulische Bildung, Friedrich-Schiller-Universität Jena, Am Planetarium 4, 07743 Jena, Deutschland

E-Mail: janine.kuhnt@uni-jena.de
} 


\title{
Tiny houses as symbols of self-realization and solidarity on the premise of self-activation
}

\begin{abstract}
Based on a qualitative content analysis of the self-presentations of selected actors inside the tiny house movement, tiny houses on the one hand can be viewed as a symbol of self-realization of a milieu, which constitutes each person's (own) norm of living due to limited resources. On the other hand, tiny houses can be viewed as an expression of solidarity with those affected by housing shortages, based on the selfactivation of engaged actors of the civil society. Both the resource-related setting of a standard of living in the smallest of spaces and the solidarity performance of engaged people with and for those affected by housing shortages make clear that this modus of engagement (alleviating housing shortages through tiny houses) is bound by conditions (available resources) and the self-activating forces of civil society actors.
\end{abstract}

Keywords Engagement · Civil society · Tiny house-movement · Housing shortage $\cdot$ Self-realization

Mitten in der Wohnraumkrise in deutschen Großstädten bewegt sich etwas. Sie sind klein, in der Regel aus Holz, mitunter auch auf Rädern stehend, weisen im Durchschnitt 11,85 qm auf und sind in der Grundausführung ihrer Erbauer*innen für durchschnittlich 42.760€ zu erwerben (vgl. Finzi und Kuhnt 2019): Tiny Houses (Winzighäuser; kurz und im Folgenden: TH). Ihre Produzent*innen wohnen entweder selbst in ihnen, bieten sie zum Verkauf an oder errichten sie mit und für Personen in Wohnungsnot. Online stellen sich die Produzent*innen selbst und ihre Produkte dar und vernetzen sich unter Hashtags wie \#tinyhousedeutschland und \#tinyhousebewegung. Neben der Selbstpräsentation, in der die Tiny-House-Produzent*innen sich als zugehörig zu einer Bewegung darstellen, kann die Zusammenfassung der unterschiedlichen Akteur*innen zu einer Tiny-House-Bewegung (kurz und im Folgenden: THB) in Anlehnung an die Definition von Graswurzelbewegungen vorgenommen werden. Hiernach sind „Grassroots (engl.: Graswurzel) [...] der gängige Ausdruck für basisorientierten Aktivismus im englischsprachigen Raum“ (Vollmer 2019, S. 233). Diese drücken sich in einer Bewegungsform aus, in der ,durch Informations- und Mobilisierungsarbeit die Durchsetzungskraft eines individuellen Partikularinteresses - einer einzigen ,Graspflanze“ - durch den Zusammenschluss mit Gleichgesinnten zu einer Gruppe - einer aus mehreren Graspflanzen bestehenden Wiese - erhöht werden kann“ (Irmisch 2013, S. 202). Dabei liegt das gemeinsame Interesse, das die Einzelpersonen der THB miteinander teilen, im Austausch über sowie der Entwicklung, Erprobung und (dauerhaften) Umsetzung des Wohnens in TH.

Einerseits wird in der THB ein zivilgesellschaftliches Potenzial deutlich:

Unter dem Begriff Zivilgesellschaft lassen sich zunächst Vereinigungen, Organisationen und soziale Bewegungen verstehen, die sich mit gesellschaftlichen Problemlagen beschäftigen. Dabei greifen sie auf, welche gesellschaftlichen Probleme im Leben der Menschen virulent werden, nehmen diese Wirkungen 
als Impulse auf, verdichten und verstärken diese und geben sie an die politische

Öffentlichkeit weiter. (Schröer et al. 2020, S. 1)

Andererseits wird mit dem TH als Wohnform eine Norm konstituiert, die aufgrund der mit ihr einhergehenden Voraussetzungen diskussionswürdig erscheint. Die THB ist eine in Deutschland bisher viel beachtete (u. a. Hauck 2021; Kramer 2021; Feireiss 2020; Lübbe 2020; Tiedge 2020; Buse 2019), aber kaum erforschte Bewegung (erste Ansätze: Maile 2020; Vollmer und Michel 2020; Kuhnt und Finzi 2019; Neupert 2018).

Dabei erfolgt die wissenschaftliche Erforschung der Bewegung aus den verschiedensten Disziplinen wie Ethnographie, Geografie und Stadtforschung sowie Erziehungswissenschaft oder der praktischen Armutsforschung. Aus einer bisher nicht beachteten, sozialpädagogischen Perspektive ist diese Bewegung insofern interessant und relevant, als mit ihr die Frage aufgeworfen wird, welches Selbst- und Hilfeverständnis die verschiedenen (zivilgesellschaftlichen) Akteur*innen der THB öffentlich kommunizieren. Die Akteur*innen der THB sind heterogen: Es können Personen sein, die ihre Wohnsituation durch den Bau und/oder Erwerb eines TH selbst gestalten, oder Personen, die durch die Bereitstellung eines TH oder einer sogenannten „Wohnbox“ (Little Home Köln e. V. 2021), die auch als „Little Home“ bezeichnet wird, Hilfeleistungen anbieten, um Wohnungsnot zu lindern. Wobei die Adressat*innen der Letztgenannten, als von Wohnungsnot Betroffene, auch Adressat*innen Sozialer Arbeit sein könnten.

Zur Erfassung der individuellen Beweggründe der Akteur*innen ist eine längerfristige qualitative Untersuchung notwendig. Fragen nach dem Selbstverständnis und der damit einhergehenden Selbstpräsentation identifizierter Akteur*innen der THB, lassen sich durch eine Analyse textlichen Kommunikationsmaterials bearbeiten, das von den Akteur*innen online präsentiert wird.

In Anlehnung an die Definition von Zivilgesellschaft und ihrer Organisationen können die Akteur*innen der THB als zivilgesellschaftliche Akteur*innen verstanden werden, die sich dem aktuell virulenten Problem „Wohnraumkrise“ widmen und in unterschiedlichen Formalisierungsgraden und Rechtsformen, Lösungen für dieses Problem entwickeln und erproben.

Dabei bewegt sich die THB in drei Spannungsfeldern, die im letzten Kapitel, basierend auf zuvor dargelegten empirischen Befunden, diskutiert werden:

1. Wohnraum ist essenziell für Teilhabe und Partizipation. Gleichzeitig kann Wohnungsnot, wenn Wohnraum nicht vorhanden ist, der Ausgangspunkt für Teilhabe und Partizipation sein.

2. Tiny Houses (TH) können Ausdruck von Selbstverantwortung engagierter Bürger*innen für die Lösung der Wohnraumknappheit und Wohnungsnot sein. Zugleich setzt dieses Engagement Ressourcen voraus, die investiert werden, um Wohnraumknappheit selbst zu lösen.

3. Mit Wohnraum in Winzighäusern werden Standards gesetzt, mit denen anerkannte Normen einerseits infrage gestellt und andererseits solche Normen geschaffen werden, die einen bestimmten Lebensstil als den angemesseneren konstituieren. 


\section{Methodisches Vorgehen}

Über online präsentierte Kommunikationsinhalte der Akteur*innen der THB sollen deren Vorstellungen und Zielstellungen, die diese mit der Erprobung und Umsetzung des Wohnens in TH verbinden, sowie ihr Hilfeverständnis erfasst werden, das im Kontext der Verfolgung der Zielstellung, Menschen in Wohnungsnot mit einem TH zu helfen, verbunden wird. Insbesondere geht es dabei um die Frage wo sich die Akteur*innen der THB in den skizzierten drei Spannungsfeldern verorten. Der Zugang zu den Selbstpräsentationen identifizierter Akteur*innen der THB basiert auf einem weiteren Verständnis von „Onlineforschung“ (Geimer 2018, S. 177). In diesem lässt sich von Onlineforschung sprechen, ,wenn sich die qualitative Forschung im Rahmen anderer Methodologien mit dem Internet auseinandersetzt (etwa Verfahren der Text-, Bild-, Filmanalyse; [...])“ (Geimer 2018, S. 177). Da zur Bearbeitung der leitenden Fragestellung nach dem Selbstverständnis der Akteur*innen der THB Kommunikationsinhalte im Fokus stehen, wurden die online zugänglichen Konzepte, Leitbilder und Visionen, die als textliches Material von den Akteur*innen öffentlich zugänglich präsentiert werden, inhaltsanalytisch ausgewertet. Die Vorteile dieses Vorgehens liegen darin, dass die online verfügbaren und für die Bearbeitung der Forschungsfrage interessierenden Kommunikationsinhalte - ohne den direkten Eingriff der Forschenden in das Forschungsfeld - bereits vorhanden sind, wenngleich die Akteur*innen selektiv nur jene Inhalte darstellen, die Auskunft darüber geben, wie sie selbst wahrgenommen werden möchten - ein limitierender Aspekt, der in der Interpretation der gewonnenen Daten berücksichtigt werden muss. Zum anderen erfordert der Onlineforschungszugang keinen langen Feldaufenthalt, der für ethnographische Ansätze typisch wäre (vgl. Geimer 2018, S. 177). Die im Sinne des Erkenntnisinteresses relevanten Kommunikationsinhalte wurden in Anlehnung an die qualitativ orientierte kategoriengeleitete Textanalyse (vgl. Mayring und Fenzl 2019, S. 634) ausgewertet und analysiert. Dabei unterscheidet sich dieser Ansatz der Textanalyse durch das Merkmal der Kategoriengeleitetheit von anderen qualitativen Ansätzen. Im Rahmen des Auswertungsprozesses wurden Kategorien zunächst induktiv, das heißt durch Paraphrasierung, Generalisierung und Reduktion relevanter Textbestandteile am Datenmaterial, gebildet. Im Anschluss daran wurden Hauptkategorien gebildet, unter denen die am Material gebildeten Kategorien als Subkategorien fungieren. Dieses Vorgehen unterscheidet sich vom methodischen Vorgehen im Rahmen einer qualitativ orientierten kategoriengeleiteten Textanalyse mittels induktiver Kategorienbildung, wie Mayring und Fenzl (2019, S. 637) es vorschlagen. Der letzte Arbeitsschritt im Prozessmodell induktiver Kategorienbildung - „Interpretation, Analyse“ - (Mayring 2015, S. 86) wurde im Zuge des Auswertungsprozesses entlang der Hauptkategorien vorgenommen, die als induktiv konstruierte Abstrahierung der zuvor gebildeten Kategorien verstanden werden können. Die Abstrahierung wird hier als induktiv konstruiert verstanden, da Kategorienbildung am Material ein aktiver Konstruktionsprozess der Forschenden ist (vgl. Kuckartz 2016, S. 72). 


\section{Sample und Kategoriensystem}

Da die THB in Deutschland aktuell zwar ein großes mediales Echo erfährt, sozialwissenschaftliche Analysen der Bewegung jedoch bisher in den Anfängen stecken, basieren die vorliegenden Befunde auf einem explorativen Vorgehen im Kontext einer Onlineforschung. Im Rahmen einer Onlinerecherche, die im Zeitraum von Januar bis August 2019 stattgefunden hat, wurden mithilfe der Suchbegriffe ,Tiny House“/,Tiny Houses“, „Winzighäuser“ und „Tiny House-Bewegung“ 14 Akteur*innen der THB identifiziert, die allesamt in das Sample aufgenommen wurden. Die identifizierten Akteur*innen stellen ein heterogenes Bild dar. Sie zeichnen sich dadurch aus, dass sie $\mathrm{TH}$ als privatwirtschaftliche Unternehmer*innen produzieren und zum Verkauf anbieten, sich über diese Wohnform in formalisierten und nichtformalisierten Zusammenschlüssen austauschen, TH als Einzelperson sowie in Gemeinschaft erproben oder explizit mittels eines Little Homes eine Hilfeleistung für Menschen in Wohnungsnot anbieten (Tab. 1).

Im Folgenden wird ein Ausschnitt des Kategoriensystems abgebildet, der die Grundlage zur Interpretation und Analyse des Selbstverständnisses der Akteur*innen der THB bildet. Dies geschieht entlang der konstruierten, induktiv aus dem Material gewonnenen Hauptkategorien HK1 Tiny Houses als Modus der Selbstverwirklichung und $H K 2$ Tiny Houses als Ausdruck von Solidarität.

Die forschungsethische Notwendigkeit der Anonymisierung der Fundstellen ergibt sich aus Sicht der Autor*innen nicht. Für die Benennung der Fundstellen spricht zum einen, dass die Kommunikationsinhalte öffentlich zugänglich sind und für die Akteur*innen selbst als „Fassaden-Seite“ (Kühl 2011, S. 92) ihrer Organisationen/ Unternehmen/Zusammenschlüsse fungieren, auf der sie ,ein schlüssiges und überzeugendes Bild ihrer selbst zu zeichnen suchen" (ebd., S. 137). Ferner geht mit der Fundstellungbezeichnung einher, dass die Forschenden dem Anspruch nach intersubjektiver Nachvollziehbarkeit nachkommen, der an qualitative Forschungsprozesse gelegt wird. Diese umfasst u. a. eine ,transparente Verfahrensdokumentation [...], in der detailliert dargestellt wird, wie die empirischen Daten erhoben und ausgewertet wurden“" (Heiser 2018, S. 48). Zumal spätestens die Eingabe wörtlicher Zitate in Online-Suchmaschinen eine Anonymisierung obsolet erscheinen lässt, da die Ergebnisse der Suchmaschinen eine Identifizierung der Akteur*innen ermöglichen.

Tab. 1 Eckdaten zu identifizierten Akteur*innen der THB. (Übernommen von Finzi und Kuhnt [2019] und leicht modifiziert)

\begin{tabular}{|c|c|}
\hline $\begin{array}{l}\text { Gründungsjahr der Rechtsform/des Zusammenschlusses } \\
\text { oder Baujahr des TH bei Einzelpersonen }\end{array}$ & $M=2016$ \\
\hline \multirow[t]{3}{*}{ Formalisierungsgrad und Rechtsformen } & $\begin{array}{l}\text { Einzelpersonen und nichtformalisierte } \\
\text { Zusammenschlüsse }=4\end{array}$ \\
\hline & Eingetragene Vereine $=2$ \\
\hline & $\begin{array}{l}\text { Privatwirtschaft (Einzelunterneh- } \\
\text { mer*innen, GmbH, GbR, UG) }=8\end{array}$ \\
\hline $\begin{array}{l}\text { Kosten für ein TH (an den Grundausführungen der Produ- } \\
\text { zent*innen orientiert) }\end{array}$ & $M=42.760 €$ \\
\hline Größe eines TH & $M=11,85 \mathrm{qm}$ \\
\hline
\end{tabular}




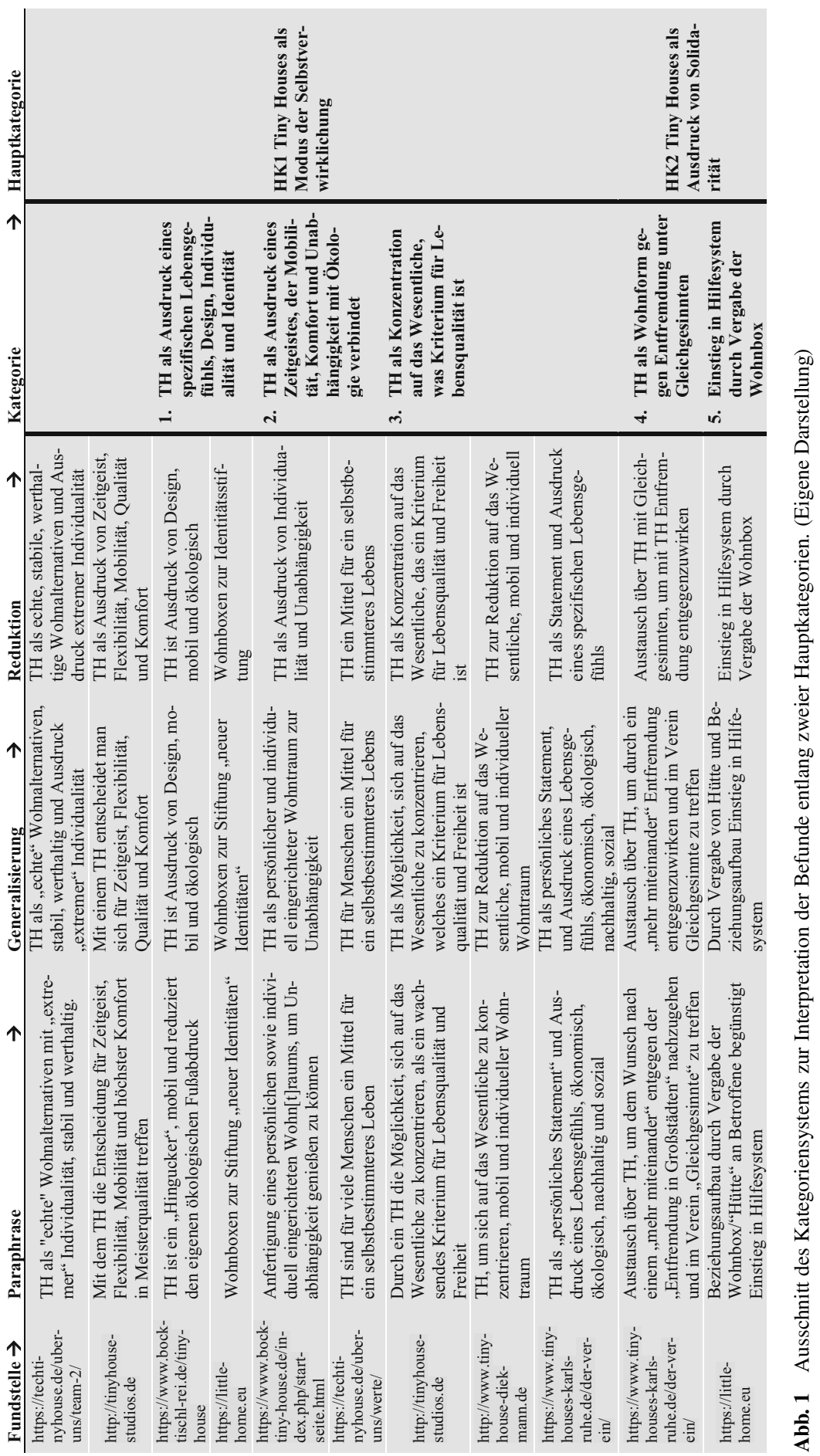


„Zugänglichkeit und Sensibilität von Inhalten sollten also in Kombination beurteilt werden“ (Schmidt 2019, S. 1023). Da es sich beim vorliegenden Material um Inhalte handelt, mit denen die Öffentlichkeit bewusst informiert, aktiviert und für den Kauf von TH, zur Unterstützung dieser Wohnform und/oder zum freiwilligen Engagement für von Wohnungsnot Betroffene aktiviert und/oder angeworben werden soll, wird die Sensibilität der Inhalte, mit der die Notwendigkeit einer Anonymisierung einherginge, als gering eingestuft.

Abb. 1 ermöglicht die intersubjektive Nachvollziehbarkeit der induktiv am Material gebildeten (Haupt-)Kategorien. Ausgehend von den Fundstellen, kann der gesamte Prozess des methodischen Vorgehens - Paraphrasierung, Generalisierung und Reduktion sowie abschließende Abstrahierung der gewonnenen Kategorien - in zwei Hauptkategorien exemplarisch erfasst werden.

\section{Tiny Houses als Modus der Selbstverwirklichung und Ausdruck von Solidarität}

Im Rahmen der Auswertung des Datenmaterials wurden fünf Kategorien am Material entwickelt: (1) Identität, (2) Zeitgeist, (3) Konzentration auf das Wesentliche, (4) Zusammenhalt und (5) Hilfsangebot. Ausgehend von diesen wurden die Hauptkategorien HK1 Tiny Houses als Modus der Selbstverwirklichung und HK2 Tiny Houses als Ausdruck von Solidarität abstrahiert. Sowohl „Selbstverwirklichung“ als auch „Solidarität“ stellen abstrakte Werte dar, die interpretationsbedürftig und zugleich aufgrund des Interpretationsspielraums gesellschaftlich anerkennt sind.

Gerade aufgrund ihres abstrakten Charakters finden gesellschaftliche Werte wie Freiheit, Gleichheit, soziale Gerechtigkeit, Solidarität und individuelle Selbstverwirklichung bei den Menschen Anerkennung als Grundlage des Zusammenlebens. Sie sind so allgemein gehalten, dass ihnen fast alle zustimmen können. Gerade deshalb können sie in konkreten Handlungssituationen unterschiedlich interpretiert werden und zur Rechtfertigung des eigenen Denkens und Handelns dienen. (Gabriel 2009, S. 31)

Wie sich die, am Material induktiv erschlossenen Werte Selbstverwirklichung und Solidarität vor dem Hintergrund eines Interpretationsspielraums einerseits und zur Rechtfertigung des eigenen Denkens und Handelns andererseits in der Selbstpräsentation der Akteur*innen der THB zeigen, wird im Folgenden dargelegt.

\subsection{Selbstverwirklichung}

Der Wert Selbstverwirklichung (Kategorien 1-3) äußert sich auf der Grundlage der Selbstpräsentationen der Akteur*innen in mehrfacher Hinsicht. Selbstverwirklichung äußert sich ...

(I) als Konzentration auf das Wesentliche - als Minimalismus -, der als Kriterium für die subjektiv empfundene Lebensqualität fungiert, 
(II) auf das Produkt TH bezogen: Das Produkt TH wird als Ausdruck eines ,Zeitgeistes“ verstanden, in dem Mobilität, Unabhängigkeit und Komfort mit ökologischen Ansprüchen verbunden werden und

(III) auf das Individuum bezogen: Durch das Leben in einem ,,individuell designten“ TH kann das Individuum einem spezifischen Lebensgefühl nachgehen, das auf den Merkmalen ökonomisch, ökologisch, nachhaltig und sozial gründet.

Selbstverwirklichung als (Selbst-)Anspruch, Prozess und Zustand, wenn diese (vermeintlich) erreicht ist, wird im sozialwissenschaftlichen Diskurs u.a. in Rekurs auf Milieu-Studien diskutiert. ${ }^{1}$ So identifizieren Barz und Liebenwein (2010,

Tab. 2 Typenbildung. (Adaptiert von Barz und Liebenwein [2010, S. 926ff.] und ergänzt durch die Wohnund Lebensform Tiny House [TH])

\begin{tabular}{|c|c|c|c|}
\hline- & $\begin{array}{l}\text { Postmaterielle } \\
\text { Das aufgeklärte Post-68er- } \\
\text { Milieu }\end{array}$ & $\begin{array}{l}\text { Moderne Performer } \\
\text { Die junge, unkonventio- } \\
\text { nelle Leistungselite }\end{array}$ & $\begin{array}{l}\text { Experimentalisten } \\
\text { Die extrem individualisti- } \\
\text { sche neue Bohème }\end{array}$ \\
\hline $\begin{array}{l}\text { Soziale } \\
\text { Lage }\end{array}$ & $\begin{array}{l}\text { Größtenteils Freiberufler/ } \\
\text { innen, Selbstständige, geho- } \\
\text { bene Angestellte und Beamte, } \\
\text { gehobene Einkommen }\end{array}$ & $\begin{array}{l}\text { Häufig Selbstständi- } \\
\text { ge, Freiberufler/innen, } \\
\text { teilweise noch in Aus- } \\
\text { bildung; gehobene } \\
\text { Einkommen }\end{array}$ & $\begin{array}{l}\text { Viele Schüler/innen und } \\
\text { Studierende; oft in freien } \\
\text { Berufen tätig; überdurch- } \\
\text { schnittliches Einkommen }\end{array}$ \\
\hline $\begin{array}{l}\text { Lebens- } \\
\text { stil/Le- } \\
\text { bens- } \\
\text { ziele }\end{array}$ & $\begin{array}{l}\text { Werte: } \\
\text { Selbstverwirklichung } \\
\text { Selbstbestimmung } \\
\text { Persönlichkeitsentfaltung } \\
\text { Umweltbewusstsein } \\
\text { Gesundheitsbewusstsein } \\
\text { Soziale Gerechtigkeit } \\
\text { Hoher Lebensstandard und } \\
\text { zugleich Aversion gegen Sta- } \\
\text { tussymbole } \\
\text { Kritische Betrachtung der } \\
\text { Globalisierungsfolgen }\end{array}$ & $\begin{array}{l}\text { Werte: } \\
\text { Großer Ehrgeiz } \\
\text { Leistungsbereitschaft } \\
\text { Selbsterprobung } \\
\text { Innovativ } \\
\text { Gegen Reglementierun- } \\
\text { gen } \\
\text { Nutzung neuer Kommu- } \\
\text { nikations- und Informa- } \\
\text { tionstechnologien } \\
\text { Idealtypus: Start-up- } \\
\text { Unternehmer }\end{array}$ & $\begin{array}{l}\text { Werte: } \\
\text { Gegen Reglementierungen } \\
\text { Gegen Hierarchien } \\
\text { Authentizität } \\
\text { Selbstverwirklichung } \\
\text { Persönlichkeitsentwicklung } \\
\text { Weltbürgerlich } \\
\text { Ausprobieren, Erfahrungen } \\
\text { sammeln: häufig gebroche- } \\
\text { ne Karriereverläufe und } \\
\text { Patchwork-Biografien }\end{array}$ \\
\hline $\begin{array}{l}\text { Wohn- } \\
\text { und } \\
\text { Le- } \\
\text { bens- } \\
\text { form } \\
\text { Tiny } \\
\text { House } \\
\text { (TH) }\end{array}$ & $\begin{array}{l}\text { TH nicht Statussymbol, son- } \\
\text { dern Symbol des gelebten } \\
\text { „Minimalismus-Mantras“ ent- } \\
\text { gegen der Norm des Wohnens } \\
\text { in größeren Eigenheimen; } \\
\text { „natürliches“ Ambiente im } \\
\text { individuell gestalteten TH (aus } \\
\text { nachhaltigen Rohstoffen) }\end{array}$ & $\begin{array}{l}\text { TH als unkonventio- } \\
\text { neller, innovativer } \\
\text { „Wohntrend“, entge- } \\
\text { gen der Norm des festen } \\
\text { Wohnsitzes; Start-up- } \\
\text { Szene privatwirtschaft- } \\
\text { licher Akteur*innen als } \\
\text { Produzierende von TH }\end{array}$ & $\begin{array}{l}\text { TH als Ausdruck ,extremer } \\
\text { Individualität“ (Tech Tiny } \\
\text { House 2019); TH als Auf- } \\
\text { begehren gegen Normen/ } \\
\text { Reglementierungen }\end{array}$ \\
\hline
\end{tabular}

\footnotetext{
1 Nicht nur auf Basis von Erkenntnissen, die durch Milieu-Studien generiert werden lässt sich das Konstrukt „Selbstverwirklichung“ anschlussfähig für die THB diskutieren. Im Rahmen seiner theoretischen Auseinandersetzungen über die Gesellschaft der Singularitäten formuliert Andreas Reckwitz (2017, S. 319; Hervorhebung: d. d. V.) aus einer soziologischen Perspektive im Hinblick auf das Wohnen bzw. die Wohnung als eine konkrete Form des Wohnens: „Die ästhetisch kuratierte Wohnung ist damit Ort performativer Selbstverwirklichung: Einerseits hat sie für Bewohner eine subjektive atmosphärische Qualität, die sie befriedigt; andererseits ist sie ein Schauplatz der Inszenierung gegenüber Besuchern, sodass ihr ästhetischer Singularitätswert für sozialen Prestige sorgt.“ Diese Performativität zeigt sich - so die Einschätzung der Autor*innen dieses Beitrages - auch in der Wohn- und Lebensform TH (Tab. 2), die durch die Bewohner*innen eines TH in unterschiedlicher Form hervorgebracht wird: TH als Symbol des gelebten ,Minimalismus-Mantras“, als innovativer „Wohntrend“ und als Ausdruck ,extremer Individualität“.
} 
S. 926ff.), auf der Basis der SINUS-Milieus und ergänzt durch weitere Studien, drei unterschiedliche Milieus, in denen Selbstverwirklichung eine bedeutende Rolle einnimmt. In Tab. 2 werden die drei unterschiedlichen milieuspezifischen Typen, die Barz und Liebenwein darlegen, in einer modifizierten Übersicht übernommen und mit der Wohn- und Lebensform TH verbunden. Beachtenswert ist dabei, dass die drei am Material entwickelten Kategorien - (I) Konzentration auf das Wesentliche, (II) Zeitgeist, (III) Identität - als Unterscheidungskriterium zwischen den Typen ausgemacht werden können.

Was alle in Tab. 2 abgebildeten milieuspezifischen Typen vereint, ist, dass sie über ein vergleichsweises hohes Einkommen und einen hohen Bildungsabschluss verfügen. Spannend ist, dass allen Typen ein Aufbegehren gegen eine, wie auch immer geartete, Norm des Wohnens zugeschrieben werden kann und dieses Aufbegehren auch in den einzelnen paraphrasierten Fundstellen des Samples (Abb. 1) ausgemacht werden kann. Die Andersartigkeit oder „,das gegen die Norm sein“ kann dabei als Alleinstellungsmerkmal oder ,unique selling proposition“ definiert werden, um einen größeren unternehmerischen Erfolg zu generieren (Homburg 2017; Nagel und Mieke 2014).

Der Modus, in dem Selbstverwirklichung im und durch ein TH angestrebt wird, gründet in der Vorstellung eines minimalistischen Lebensstils als den im Verhältnis zu anderen, der Norm entsprechenden Lebensstilen angemesseneren. Die kritisch zu betrachtenden Begründungsmotive für eine auf Minimalismus ruhende Form der Selbstverwirklichung, die den Selbstdarstellungen der Akteur*innen zu entnehmen sind, reichen von (I) einem sparsamen Umgang mit Ressourcen/Rohstoffen im Sinne eines individuellen und gesellschaftlichen Benefits (hierzu auch kritisch: Kuhnt und Finzi in Vorbereitung), über (II) ein Trendphänomen, dem eine junge ,Start-upSzene“ (auch) aus ökonomischen Beweggründen nacheifert, bis (III) zur Möglichkeit des Erprobens reduzierten und zugleich extrem individuellen Wohnens, auf der Suche nach Authentizität. Authentizität wird hier ,zu einem machtvollen Werkzeug, das sich gegen alle richtet, die diese Semiotisierung nicht nachvollziehen können oder wollen. (...) Die ausgeübte Macht über den Raum ist sowohl eine finanzielle als auch eine kulturelle“ (Vollmer 2019, S. 94). Sowohl die kulturelle als auch die finanzielle Macht manifestiert sich im TH nicht vordergründig als objektiv gegebenes Produkt, sondern vor allem als ,Statement“ einer die Normen des Wohnens infrage stellenden Bewegung, die im TH als Zeichenträger selbst Normen setzt (u. a. mobiles Wohnen in einem Kleinst-Eigentum, das den eigenen Ansprüchen an Individualität, Komfort und Nachhaltigkeit entspricht).

Wohnraum ist für uns mehr als eine Aufbewahrungsstätte für Menschen und Besitz. Er ist ein persönliches Statement, Ausdruck eines Lebensgefühls und unmittelbar mit Themen wie Nachhaltigkeit, Ökonomie, Ökologie, Sozialem und Kultur verknüpft. (Tiny Houses für Karlsruhe e. V. 2019)

\subsection{Solidarität}

In den Selbstpräsentationen der Akteur*innen und den Darstellungen ihrer Visionen zeigt sich neben der primär selbstbezüglichen Vorstellung von TH als Modus 
der Selbstverwirklichung eine Vorstellung von TH als solidarische Ausformung der THB, die primär auf einen Fremdnutzen ${ }^{2}$ gerichtet wird und ein zivilgesellschaftliches Potenzial bergt. Ein primär (aber nicht ausschließlich) auf den Fremdnutzen ausgerichtetes Selbstverständnis, mit dem TH als Wohnform entwickelt, produziert und erprobt werden, lässt sich im vorliegenden Sample insbesondere bei Akteur*innen finden, die in der Rechtsform des eingetragenen Vereins organisiert sind und sich insofern der organisierten Zivilgesellschaft oder synonym dem Dritten Sektor zuordnen lassen.

Im Dritten Sektor ist die Ressource „Solidarität“ eine treibende Kraft - sowohl als Motivationsgrund der Mitglieder, der Engagierten und der Förderer als auch als Leitmotiv und Medium der Handlungskoordination seiner Organisationen. (Liebig und Rauschenbach 2010, S. 261)

Die Ressource Solidarität zeigt sich im Selbstverständnis der in Vereinen organsierten Akteur*innen der THB in zweifacher Hinsicht. Zum einen zeigt sie sich als Solidarität mit „Gleichgesinnte[n]“ (Tiny Houses für Karlsruhe e. V. 2019), das heißt in Bezug auf an dieser spezifischen Wohnform Interessierte und Vereinsmitglieder, die die gemeinsame Vision verfolgen, mit der Wohnform TH der gesellschaftlichen Herausforderung zu begegnen, Lösungen für knapper und teurer werdenden Wohnraum zu schaffen ${ }^{3}$.

Knapper und immer teurer werdender Wohnraum sorgt dafür, dass sich viele Menschen Gedanken über alternative Wohnformen machen. Gleichzeitig erzeugt die Entfremdung der Menschen in der Großstadt den Wunsch, mehr miteinander als nebeneinander her [sic!] zu leben. Verspürst Du diesen Wunsch auch? Und machst auch Du Dir Gedanken über das Wohnen auf kleinem Raum? Wenn ja, dann bist Du bei uns genau richtig. Egal, ob Kleinraumwohnen mit oder ohne Räder - der Verein „Tiny Houses für Karlsruhe“ ist Deine Anlaufstelle, um Gleichgesinnte zu treffen. (Tiny Houses für Karlsruhe e. V. 2019)

Zum anderen zeigt sie sich als Solidarität mit von Wohnungsnot Betroffenen, für die Wohnboxen auf der Basis von Spenden und den Mitgliedsbeiträgen der Engagierten, die sich in der Rechtsform des eingetragenen Vereins organisierten, gebaut werden.

Durch ein Leben im Little Home ist diese kleine Gruppe von Menschen, die ansonsten obdachlos auf der Straße leben würde, ein (Über-)Lebensraum sowie ein erster Einstieg in weitere Hilfen. [...] Vor allem bietet LITTLE HOME

\footnotetext{
${ }^{2}$ Der Fremdnutzen ist in der THB v. a. auf den Gebrauch des Produktes TH gerichtet; das Produkt wird entweder durch Schenkung oder Kauf erworben. Während Erstgenanntes im Fortlauf dieses Textes diskutiert wird, muss hinsichtlich des Fremdnutzens durch Kauf und Verkauf von TH auf einen anderen Beitrag verwiesen werden, in dem Tiny Houses als „Renditeobjekte“ diskutiert werden (vgl. Kuhnt und Finzi 2021).

3 Diese Frage der Flächengerechtigkeit wird bereits in einer Antwort auf den Debattenaufruf von Vollmer und Michel (2020), durch Brokow-Loga und Neßler (2020), als auch in der aktuellen Debatte um das Verbot des Baus von Eigenheimen (Twickel 2021) diskutiert. Beachtenswert für die THB ist, dass diese einen offensichtlichen Flächenverbrauch als eine mögliche Lösung darstellt und nicht kritisch hinterfragt.
} 
diesen Menschen das Mindestmaß an Privatsphäre, die ein Grundbedürfnis eines jeden Menschen ist. (GRUNDBEDÜRFNISSE!!) Deshalb können sie sich gut auf dieses Angebot einlassen und sind in ihren Hütten für aufsuchende Soziale Arbeit regelmäßig und verlässlich erreichbar. Dadurch kann es gelingen, tragfähige Beziehungen aufzubauen und die Menschen so in weitere Hilfen zu vermitteln, um deren bestehende soziale Schwierigkeiten zu bearbeiten. (Little Home Köln e. V. 2019; Hervorhebung i. O.)

In beiden Formen geht Solidarität aus dem Selbstverständnis und freiwilligem Engagement von Unterstützer*innen und Vereinsmitgliedern hervor, deren Aktivitäten primär von einem Fremd- aber auch von einem Selbstbezug gekennzeichnet sind.

Bürgerschaftliches Engagement muss positive Effekte für Dritte aufweisen, also Gemeinwohlbezug haben. Das bedeutet aber nicht, dass nur altruistische Motive im Vordergrund stehen müssen, ebenso kann die Motivation zum bürgerschaftlichen Engagement einen Selbstbezug (z.B. Selbstverwirklichung) haben oder Formen der Selbsthilfe umfassen. (Schmid 2010, S. 353)

Der Gemeinwohlbezug auf der Basis von Solidarität mit Dritten wird in der THB zum einen in der Vision deutlich, als organisierter Zusammenschluss von Engagierten ein gesellschaftlich relevantes Problem lösen zu wollen (Wohnraumknappheit und Wohnungsnot) oder zumindest eine Wohnform zu erproben, die zu dessen Lösung beitragen soll. Darüber hinaus geht Solidarität in dieser Bewegung an jenem Punkt über die Vertretung primär eigener Interessen hinaus, an dem es um die gezielte Minderung menschlichen Leidens durch Wohnungsnot geht. Hier wird engagiertes Handeln, das darauf ausgelegt ist, Menschen durch die Vergabe einer Wohnbox, einen Einstieg in das Hilfesystem zu ermöglichen, zum einen als Ausdruck kultureller und finanzieller Macht: Die Helfenden agieren aus einer Position heraus, die ihnen durch vorhandene oder generierte Ressourcen ermöglicht wird und in der sie über Vergabe und Nichtvergabe einer Wohnbox entscheiden können. Zum anderen ist diese Form des Engagements - das auf Solidarität beruht - ein Statement, in dem Eigensinn einen Ort findet. „Helfen wäre dann solidarisch, wenn es eine Verbindung dieses jeweiligen Eigensinns untereinander leistet, was nichts anderes bedeutet, als Menschen durch Helfen zum Handeln anzustiften" (Weber 2019, S. 29). Eigensinn drückt sich zum einen in der Form des Engagements aus, indem freiwillig Engagierte sich organisieren, um Little Homes (mit einer Größe von 3,2 qm) zu produzieren und an Menschen in Wohnungsnot zu vergeben. Diese Hilfeform weicht von den Normen im Hilfesystem ab: Die Größe droht die hart erkämpften Wohnstandards zu senken (Neupert 2018), außerdem unterläuft das Engagement die Unterbringungspflicht der Kommunen (Rosenke 2017). Zugleich wird durch die eigensinnige Form des Helfens selbst eine Norm konstruiert: eine Norm, im Rahmen derer dieser Winzig-Wohnraum als für von Wohnungsnot Betroffene angemessene Hilfe- und Wohnform konzipiert wird. Überdies äußert sich Eigensinn potenziell auch auf Seiten der Hilfebedürftigen, wenn diese Hilfe ablehnen und/ oder mitgestalten und/oder annehmen. Eigensinn in diesem Sinne führt zurück zu den eingangs skizzierten Spannungsfeldern, die abschließend diskutiert werden. 


\section{TH als symbolisierter Eigensinn zivilgesellschaftlicher Akteur*innen}

Die in der Einleitung vorgestellten drei Spannungsfelder - Teilhabe, Selbstverantwortung und Normen - werden im Folgenden und basierend auf den vorgestellten empirischen Befunden kritisch diskutiert.

1. Wohnraum, als Schutzraum aber auch als Gestaltungsraum, ist essenziell für Teilhabe und Partizipation (Gerull 2018; Spellerberg und Giehl 2018; Gillich und Nieslony 2000; Finzi in Vorbereitung), und gleichzeitig kann Wohnungsnot, also das Nichtvorhandensein dieser essenziellen Voraussetzung für Teilhabe, deren Ausgangspunkt sein. Dass Wohnraum essenziell für Teilhabe ist, lässt sich über das Selbstverständnis und die Funktion Sozialer Arbeit ableiten, deren professioneller Auftrag darin besteht, dass sie ihre Klient*innen dazu befähigt, ihre Rechte (hier das Recht auf Wohnraum) selbstständig wahrnehmen und realisieren zu können (im Sinne der Hilfe zur Selbsthilfe ${ }^{4}$ ) und um insofern ihrer Funktion sozialer Integration nachzukommen (vgl. Merten 2001, S. 91, 98). Ferner können ihre Hilfen ,oft nur dann erfolgreich sein und abgeschlossen werden, wenn eine Vermittlung in angemessenen Wohnraum gelingt" (Nagel 2013, S. 19). In diesem Sinne ermöglichen die Vereine der THB, unter Berücksichtigung ihres auf Solidarität gründenden Selbstverständnisses, (Bei-)Hilfe zur sozialen Inklusion. Sie stellen Hilfebedürftigen oder Adressat*innen, die (noch) nicht Betroffene, aber Engagement-Interessierte sind, ein Angebot bereit, damit diese ihre Rechte selbstständig wahrnehmen oder an der Entwicklung von Lösungsansätzen zur Wahrnehmung ihres Rechts teilhaben können. Inwiefern jedoch solche Wohnboxen auch gegenteilige, also exkludierende und stigmatisierende Effekte haben können - was durchaus naheliegend ist -, wird im Zuge von (2) das TH als Zeichenträger beleuchtet.

Zugleich ist ein konstitutives Element des Engagements mit Selbst- und Fremdbezug Betroffenheit; ich konstituiere mich oder andere als Betroffene, die zur Selbstund Mithilfe aktiviert werden. Die Voraussetzung für diese Form des Engagements und der Selbsthilfe ist die Investition unterschiedlicher Ressourcen (finanzielle, persönliche und soziale), um Mitglied eines organisierten Zusammenschlusses und Kund*in zu werden oder um Hilfebedarfe bedienen zu können.

2. TH können Produkt und Zeichenträger von Selbstverantwortung engagierter Bürger*innen für die Lösung von Wohnraumknappheit und Wohnungsnot sein. Zugleich setzt dieses Engagement Ressourcen sowie Solidarität mit Betroffenen voraus, um Wohnraumknappheit selbst zu lösen. Was durch die voraussetzungsvollen Bedingungen und Implikationen des Wohnens in TH nicht gelöst wird, sind soziale Ungleichheiten, die im Zeichenträger TH eher verstetigt werden $(\rightarrow$ siehe Typen in Tab. 2). „In diesem Zusammenhang sollte sie [Soziale Arbeit; d. V.] sich kritisch dazu verhalten, was als ,angemessenerer Wohnraum' definiert wird

\footnotetext{
4 „Sozialraumorientiertes Handeln impliziert die Aktivierung, Bündelung und Koordinierung aller Kräfte, die Teilhabe ermöglichen. [...] Sozialraumorientierung beinhaltet das Schaffen grundlegender Strukturen, die Hilfe und Selbsthilfe ermöglichen. Auf der subjektiven Ebene ist ein Arbeitsauftrag der Wohnungslosenhilfe, die Anliegen und Interessen der Klientel zu vertreten. Der sozialraumorientierte Handlungsansatz beinhaltet die Ermutigung und Unterstützung der Hilfesuchenden zu einer selbstständigen Interessens- und Anliegenvertretung.“ (Frölich und Keim 2012, S. 112).
} 
und inwiefern es legitim erscheint, dass strukturelle Problemlagen $\mathrm{zu}$ - durch eine aktivierte Zivilgesellschaft - selbstverantwortlich zu lösende Problemlagen werden“ (Kuhnt 2020). Mit der Vergabe eines TH an einen von Wohnungsnot Betroffenen durch professionelle Akteur*innen eines Wohlfahrtverbandes, mit dem Ziel verbunden, hierdurch ein Zeichen für mehr privates Engagement durch Zivilgesellschaft und Unternehmen zu setzen (vgl. Evangelisch-Lutherische Landeskirche Hannovers 2019), wird die Problemlage Wohnungsnot und deren Lösung primär individualisiert und weniger - im Sinne politischer Interessenvertretung auf ein sozialpolitisches Terrain gehoben. Diese Individualisierung ist seit jeher Kernelement der Abwertung und Stigmatisierung von Wohnungsnot (Finzi in Vorbereitung; Teidelbaum 2020). Darüber hinaus kann neben der Individualisierung die Sichtbarmachung durch eine Wohnbox respektive eines TH die bereits hoch vulnerable und stigmatisierte Gruppe der Personen in Wohnungsnot neuen Stigmatisierungen aussetzen. Wohnungsnot, Wohnungslosigkeit und Obdachlosigkeit werden in besonderem Maße stigmatisiert (Busch-Geertsema et al. 2019, S. 141), daher versuchen Menschen in Wohnungsnot unsichtbar zu werden, nicht aufzufallen und sich zu verstecken (Wolf 2016, S. 9f.). Wohnboxen machen Wohnungsnot jedoch deutlich sichtbar. Sie zeigen, dass diejenige Peron, die in einer solch kleinen Wohnbox schlafen muss, die Bewältigung ihrer sozialen Problemlage nicht ohne Hilfe durch Dritte geschafft hat. Personen in dieser sozialen Problemlage brauchen, so wird im TH als Symbol solidarischer Hilfeleistung deutlich, offensichtlich Hilfe zur Bewältigung einer Problemlage, die andere selbst bewältigen können. Der (Trug-)Schluss, dass dem Individuum eine eigene Schuld, ein eigenes Versagen für die Lebenssituation zugeschrieben wird, ist schnell gefasst und führt zu einer weiteren Abwertung und Stigmatisierung in der meritokratischen Leistungsgesellschaft und möglicherweise einer noch größeren Scham für Betroffene (Gerull 2018).

Aus der Perspektive eines wohlsituierten gesellschaftlichen Milieus, das nicht von einer existenziellen Dringlichkeit getrieben auf die Schaffung und Bereitstellung von Wohnraum angewiesen ist, sondern sich - leitend von den milieuspezifischen Vorstellungen eines als legitim erachteten minimalistischen Lebensstils für den Erwerb eines TH entscheiden kann und als Kund*innen mit Kaufkraft die Wahl hat, mag die Selbstaktivierung Freiheit im Sinne von Selbstbestimmung und Selbstverantwortung bedeuten. Angenommen werden kann, dass das TH (v. a. das Little Home) aus der Perspektive eines Menschen in Wohnungsnot wohl eher ein sichtbares Symbol - ein Stigma - (i) für die Angewiesenheit auf Solidaritätsleistungen durch Dritte, (ii) die Unfähigkeit, sich auf dem Wohnungsmarkt selbst zu helfen, und (iii) die Akzeptanz einer Übergangslösung ist, verbunden mit der Hoffnung des Einstiegs in das soziale Hilfesystem. Wo doch Menschen in Wohnungsnot sich zumeist unsichtbar machen, um keinen Abwertungen und Stigmatisierungen ausgesetzt zu sein (Gerull 2018; Wolf 2016). Inwiefern TH hilfreich für den Einstieg in weiterführende Hilfen sind, kann und soll an dieser Stelle nicht beantwortet werden.

3. Zugleich werden durch diese Form des Engagements mit Selbst- und Fremdbezug, durch die Produktion, den Kauf und/oder Konsum von Wohnraum in Winzighäusern nicht nur Normen infrage gestellt, sondern auch solche geschaffen, die 
einen bestimmten Lebensstil als den angemesseneren konstituieren oder Selbsthilfe in Graswurzelbewegungen als legitime Lösung gesellschaftlicher Probleme erscheinen lassen. ,In addition to alternative projects with a pronounced middle-class character, the grassroots renaissance of community is often rooted in the breakdown of public infrastructure“ (van Dyk 2018, S. 536). So wären im OfflineModus der Entstehungskontext der THB und ihr integratives Potenzial zu untersuchen, deuten ja gerade die voraussetzungsvollen Bedingungen dieses Engagements auf einen „middle-class character“ und einen latenten Zusammenbruch der öffentlichen Infrastruktur (in diesem Bereich sozialer Daseinsvorsorge) hin, durch den Hilfebedürftige auf ihre Selbstaktivierungskräfte verwiesen werden oder auf diese angewiesen (Solidarität als durch Helfen zum Handeln anstiften) sind.

Bereits die kurze Abhandlung der skizzierten Spannungsfelder verdeutlicht, dass es im sozialpädagogischen Handlungsfeld der Hilfe für Menschen in Wohnungsnot im Zusammenhang mit der THB - insbesondere qualitative - Forschungsbedarfe gibt (u.a. Dittmann und Drilling 2018). Viele Fragen - die Debatte um den ökologischen Effekt, mögliche negative Effekte dieses solidarischen Handelns, aber auch die Tatsache, dass ein TH und dessen Bau voraussetzungsvoll sind - sind weiterhin offen und könnten bei genauerer Betrachtung eher nichtintendierte Effekte nach sich ziehen. Auch ist nicht geklärt, inwieweit sich die Start-up-Unternehmen in der Bewegung als „Social Entrepreneurs“ betrachten, die zur Lösung eines gesellschaftlichen Problems beitragen (Kuhnt und Finzi in Vorbereitung). Anschlussfragen sind überdies, wie sich die Professionellen (u. a. Sozialpädagog*innen) und ihre Organisationen (z.B. die Wohlfahrtsverbände) zu dieser jungen Bewegung und ihren Ausformungen verhalten, welche Herausforderungen sich im Kontext der Aufgabenund Verantwortungsteilung zur Bereitstellung von Wohnraum zwischen Kommune, privaten und privatwirtschaftlichen Akteur*innen aus der Perspektive der Engagierten in dieser Bewegung stellen und zu welchen Einschätzungen alle Akteur*innen über diese Hilfeform von Wohnungsnot zukünftig gelangen.

Die THB schafft durch ihren Resonanzraum eine große (insbesondere mediale) Aufmerksamkeit und somit das Potenzial, tatsächliche Veränderungen anzustoßen. Wohin diese allerdings führen und ob die Verkaufsargumente eher solche bleiben oder zu tatsächlichen Veränderungen führen, ist offen. Auch wenn sich ihre Akteur*innen als Bewegung formieren, ist ungeklärt, wohin sich diese zukünftig bewegen.

Funding Open Access funding enabled and organized by Projekt DEAL.

Open Access Dieser Artikel wird unter der Creative Commons Namensnennung 4.0 International Lizenz veröffentlicht, welche die Nutzung, Vervielfältigung, Bearbeitung, Verbreitung und Wiedergabe in jeglichem Medium und Format erlaubt, sofern Sie den/die ursprünglichen Autor(en) und die Quelle ordnungsgemäß nennen, einen Link zur Creative Commons Lizenz beifügen und angeben, ob Änderungen vorgenommen wurden.

Die in diesem Artikel enthaltenen Bilder und sonstiges Drittmaterial unterliegen ebenfalls der genannten Creative Commons Lizenz, sofern sich aus der Abbildungslegende nichts anderes ergibt. Sofern das betreffende Material nicht unter der genannten Creative Commons Lizenz steht und die betreffende Handlung 
nicht nach gesetzlichen Vorschriften erlaubt ist, ist für die oben aufgeführten Weiterverwendungen des Materials die Einwilligung des jeweiligen Rechteinhabers einzuholen.

Weitere Details zur Lizenz entnehmen Sie bitte der Lizenzinformation auf http://creativecommons.org/ licenses/by/4.0/deed.de.

\section{Literatur}

Barz, H., \& Liebenwein, S. (2010). Kultur und Lebensstile. In R. Tippelt \& B. Schmidt (Hrsg.), Handbuch Bildungsforschung (Bd. 3, S. 915-936). Wiesbaden: VS.

Brokow-Loga, A., \& Neßler, M. (2020). Eine Frage der Flächengerechtigkeit. Sub\urban. Zeitschrift Für Kritische Stadtforschung, 8(1/2), 183-192. https://doi.org/10.36900/suburban.v8i1/2.552.

Busch-Geertsema, V., Henke, J., \& Steffen, A. (2019). Entstehung, Verlauf und Struktur von Wohnungslosigkeit und Strategien zu ihrer Vermeidung und Behebung. Ergebnisbericht - Forschungsbericht, Bd. 534. Bremen: Gesellschaft für innovative Sozialforschung und Sozialplanung e.V..

Buse, U. (2019). Wir hätten gern das Sofa behalten, aber das ging nicht. https://www.spiegel.de/panorama/ tiny-houses-wie-viel-platz-braucht-man-zum-leben-a-00000000-0002-0001-0000-000166979776. Zugegriffen: 3. Juni 2021.

Dittmann, J., \& Drilling, M. (2018). Armut und Wohnungslosigkeit. In P. Böhnke, J. Dittmann \& J. Goebel (Hrsg.), Handbuch Armut: Ursachen, Trends, Maßnahmen (Bd. 4957, S. 282-293). Opladen: Barbara Budrich.

van Dyk, S. (2018). Post-wage politics and the rise of community capitalism. Work, Employment and Society, 32(3), 528-545.

Evangelisch-Lutherische Landeskirche Hannovers (2019). Es ist ein Zeichen! https://www.landeskirchehannovers.de/evlka-de/presse-und-medien/frontnews/2019/01/24. Zugegriffen: 11. Febr. 2020.

Feireiss, N. (2020). Das Eigenheim für Wohlstandspunks. https://www.zeit.de/wirtschaft/2020-12/ minihaeuser-tiny-houses-trend-minimalismus-wohnen-lebensweise. Zugegriffen: 6. März 2021.

Finzi, J., \& Kuhnt, J. (2019). Die Tiny House-Bewegung als Akteurin der Zivilgesellschaft - Eine Inhaltsanalyse ihres Selbstverständnisses. In Soziale und politische Ungleichheit? Interessenvertretung in der Sozialpolitik. Tagung der Sektion Sozialpolitik in der DGS und des AK Wohlfahrtsstaatsforschung in der DVPW, Universität Essen, 10.-11.10.2019.

Finzi, J. (i.E.). Wohnungsnot, Geschlecht und Gesundheit. Eine Analyse von Teilhabe und Stigmatisierung. Dissertation, Technische Universität Dortmund.

Frölich, N., \& Keim, R. (2012). Gemeinwesenperspektiven in der ambulanten Wohnungslosenhilfe. In S. Gillich \& R. Keicher (Hrsg.), Bürger oder Bettler (S. 107-117). Wiesbaden: VS.

Gabriel, O.W. (2009). Politische Kultur. In K. Viktoria \& A. Römmele (Hrsg.), Politische Soziologie (S. 17-51). Wiesbaden: VS.

Geimer, A. (2018). Onlineforschung. In R. Bohnsack, A. Geimer \& M. Meuser (Hrsg.), Hauptbegriffe Qualitativer Sozialforschung (S. 175-179). Opladen, Toronto: Verlag Barbara Budrich.

Gerull, S. (2018). „Unangenehm“, „Arbeitsscheu“, „Asozial“ - Zur Ausgrenzung von wohnungslosen Menschen. Aus Politik Und Zeitgeschichte, 68(25-26), 30-36. Die Ausgrenzung wohnungsloser Menschen hat eine lange Tradition in Deutschland. Auch heutzutage sind sie massiven Ausgrenzungs- und Stigmatisierungsprozessen ausgesetzt, die in Gewalt gegen diese vermeintlich homogene Gruppe eskalieren können.

Gillich, S., \& Nieslony, F. (2000). Armut und Wohnungslosigkeit: Grundlagen, Zusammenhänge und Erscheinungsformen. Reihe soziale Arbeit, Bd. No. F 0057. Köln: Fortis.

Hauck, S. (2021). Große Freiheit in kleinem Haus. https://www.sueddeutsche.de/muenchen/wolfratshausenbarbara-lexa-kuenstlerin-1.5174792. Zugegriffen: 6. März 2021.

Heiser, P. (2018). Meilensteine der qualitativen Sozialforschung: eine Einführung entlang klassischer Studien. Wiesbaden: Springer VS.

Homburg, C. (2017). Marketingmanagement: Strategie - Instrumente - Umsetzung - Unternehmensführung. Bd. 6. Wiesbaden: Springer VS. https://doi.org/10.1007/978-3-658-13656-7.

Irmisch, A. (2013). Graswurzelkommunikation im Kontext politischer Interessenvertretung. In R. Speth (Hrsg.), Grassroots-Campaigning (S. 201-212). Wiesbaden: Springer VS.

Kramer, H. (2021). Leben auf 33 Quadratmetern. https://www.sueddeutsche.de/muenchen/dachau/dachautiny-house- wohnform-altomuenster-1.5202363?reduced=true. Zugegriffen: 6. März 2021.

Kuckartz, U. (2016). Qualitative Inhaltsanalyse. Methoden, Praxis, Computerunterstützung (3. Aufl.). Weinheim, Basel: Beltz Juventa. 
Kühl, S. (2011). Organisationen. Wiesbaden: VS.

Kuhnt, J. (2020). Wohnungsnot minimalistisch, selbstverantwortlich und/oder sozialarbeiterisch angehen? Blätter der Wohlfahrtspflege, 167(2), 53-55.

Kuhnt, J., \& Finzi, J. (2021). Weniger Haus, mehr Leben(squalität)?! Die Tiny House-Bewegung zwischen Revolte und Rendite. In M. Staats (Hrsg.), Lebensqualität. Ein Metathema. Weinheim: Beltz Juventa.

Kuhnt, J., \& Finzi, J. (i.E.). Selbst-Inszenierungen über das Narrativ Nachhaltigkeit: Wie Tiny HouseProduzent*innen als Sozial- und Selbst-Unternehmer*innen online „Nachhaltigkeit“ erzählen. In A. Bush \& J. Birke (Hrsg.), Nachhaltigkeit und Social Media. Bildung für eine nachhaltige Entwicklung in der digitalen Welt. Wiesbaden: Springer VS.

Liebig, R., \& Rauschenbach, T. (2010). Die engagementpolitische Rolle von Akteuren des Dritten Sektors. In T. Olk, A. Klein \& B. Hartnuß (Hrsg.), Engagementpolitik. Die Entwicklung der Zivilgesellschaft als politische Aufgabe (S. 260-281). Wiesbaden: VS.

Little Home Köln e. V. (2019). Warum Little Home. https://little-home.eu. Zugegriffen: 19. Sept. 2019.

Little Home Köln e. V. (2021). Die Little Homes. https://little-home.eu. Zugegriffen: 10. März 2021.

Lübbe, S. (2020). 35 Euro für den Quadratmeter Land. https://www.zeit.de/wirtschaft/2020-12/stadtfluchtcorona-pandemie-landleben-infrastruktur-verkehrsanbindung-schule. Zugegriffen: 3. Juni 2021.

Maile, L. (2020). Tiny house movement: Alternative Wohn- und Lebensformen als Ausdruck einer Postwachstumsstrategie. Münchner ethnographische Schriften. München: utzverlag GmbH.

Mayring, P. (2015). Qualitative Inhaltsanalyse. Grundlagen und Techniken (12. Aufl.). Weinheim, Basel: Beltz.

Mayring, P., \& Fenzl, T. (2019). Qualitative Inhaltsanalyse. In N. Baur \& J. Blasius (Hrsg.), Handbuch Methoden der empirischen Sozialforschung (S. 633-648). Wiesbaden: Springer.

Merten, R. (2001). Politisches Mandat als (Selbst-)Missverständnis des professionellen Auftrags Sozialer Arbeit. In R. Merten (Hrsg.), Hat Soziale Arbeit ein politisches Mandat? Positionen zu einem strittigen Thema (S. 89-100). Wiesbaden: Springer.

Nagel, S. (2013). Ausgrenzung und Diskriminierung auf dem Wohnungsmarkt. Widersprüche, 127(1), 9-21.

Nagel, M., \& Mieke, C. (2014). BWL-Methoden: Handbuch für Studium und Praxis. UTB Betriebswirtschaftslehre, Bd. 8564. Konstanz: UVK. Retrieved from http://www.utb-studi-e-book.de/ 9783838585642

Neupert, P. (2018). Leben im Tiny House - ein geeignetes Instrument gegen Wohnungsnot? Wohnungslos, $60(1), 14-19$.

Reckwitz, A. (2017). Die Gesellschaft der Singularitäten. Zum Strukturwandel der Moderne. Berlin: Suhrkamp.

Rosenke, W. (2017). Integrierte Notversorgung. In Bundesarbeitsgemeinschaft Wohnungslosenhilfe e. V. (Hrsg.), Handbuch der Hilfen in Wohnungsnotfällen: Entwicklung lokaler Hilfesysteme und lebensbezogener Hilfeansätze (S. 203-217). Berlin, Düsseldorf: Bundesarbeitsgemeinschaft Wohnungslosenhilfe e. V..

Schmid, J. (2010). Engagementpolitik auf Landesebene - Genese und Strukturierung eines Politikfeldes. In T. Olk, A. Klein \& B. Hartnuß (Hrsg.), Engagementpolitik. Die Entwicklung der Zivilgesellschaft als politische Aufgabe (S. 352-381). Wiesbaden: VS.

Schmidt, J.-H. (2019). Blogs. In N. Baur \& J. Blasius (Hrsg.), Handbuch Methoden der empirischen Sozialforschung (S. 1015-1025). Wiesbaden: Springer.

Schröer, A., Engel, N., Fahrenwald, C., Göhlich, M., Schröder, C., \& Weber, S. M. (2020). Organisation und Zivilgesellschaft. Einführung. In A. Schröer, N. Engel, C. Fahrenwald, M. Göhlich, C. Schröder \& S. M. Weber (Hrsg.), Organisation und Zivilgesellschaft (S. 1-8). Wiesbaden: Springer VS.

Spellerberg, A., \& Giehl, C. (2018). Armut und Wohnen. In P. Böhnke, J. Dittmann \& J. Goebel (Hrsg.), Handbuch Armut: Ursachen, Trends, Maßnahmen. UTB Sozialwissenschaften, (Bd. 4957, S. 270-281). Opladen, Toronto: Barbara Budrich.

Teidelbaum, L. (2020). Sozialdarwinismus: Die Abwertung von (vermeintlich) Schwächeren. Wohnungslos, 62(2), 38-40.

Tiedge, A. (2020). Tiny House auf Rädern. https://www.spiegel.de/reise/im-schulbus-unterwegs-soloreisende-und-familie-erzaehlen-von-erfahrungen-mit-skoolie-a-0da53ad7-8e36-4119-86a20447ca8581f1. Zugegriffen: 6. März 2021.

Tiny Houses für Karlsruhe e. V. (2019). Der Verein. https://www.tiny-houses-karlsruhe.de/der-verein/. Zugegriffen: 19. Sept. 2019.

Twickel, C. (2021). Es geht nicht darum, Menschen vorzuschreiben, wie sie leben sollen. https://www. zeit.de/hamburg/2021-02/einfamilienhaeuser-hamburg-verbot-wohnraum-flaechen-umwelt. Zugegriffen: 13. März 2021. 
Vollmer, L. (2019). Mieter_innenbewegungen in Berlin und New York: Die Formierung politischer Kollektivität. Wiesbaden: Springer.

Vollmer, L., \& Michel, B. (2020). Wohnen in der Klimakrise. Sub\urban. Zeitschrift Für Kritische Stadtforschung, 8(1/2), 163-166. https://doi.org/10.36900/suburban.v8i1/2.552.

Weber, J. (2019). Kritik der Solidarität. Widersprüche, 151(1), 19-31.

Wolf, S. (2016). Über die Wahrnehmung von und den Umgang mit obdachlosen Personen im öffentlichen Raum. Weimar: Katholische Arbeitsgemeinschaft Wohnungslosenhilfe. 